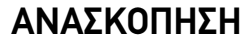

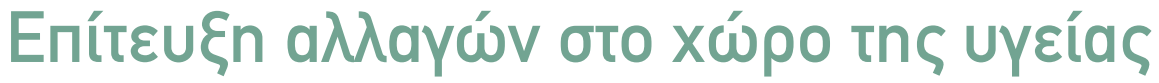

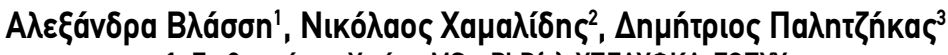

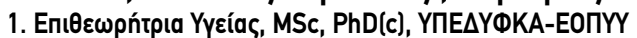

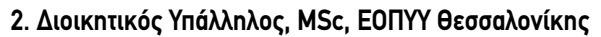

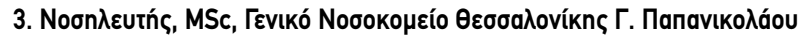

\begin{abstract}
ПEPIAHUH

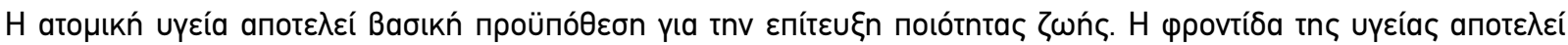

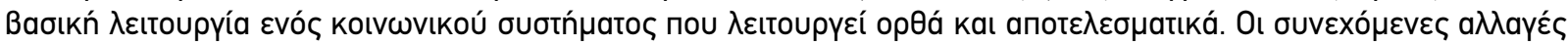

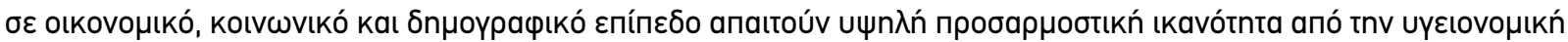

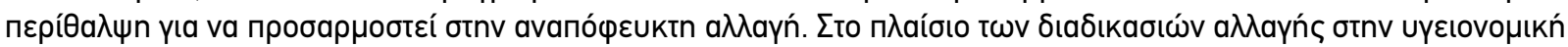

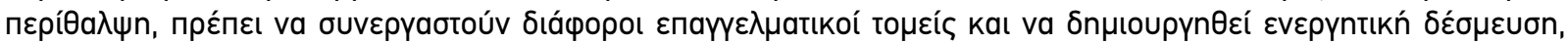

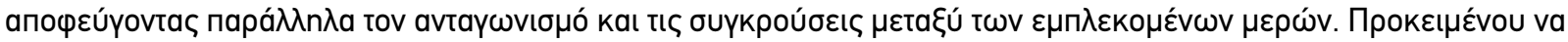

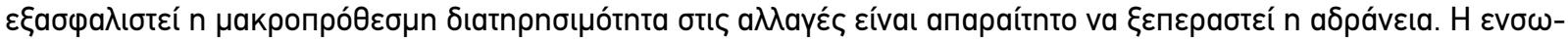

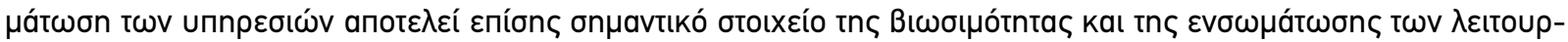

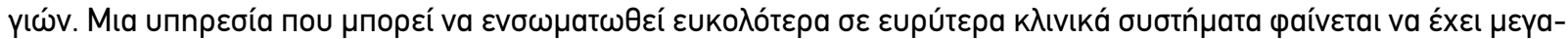

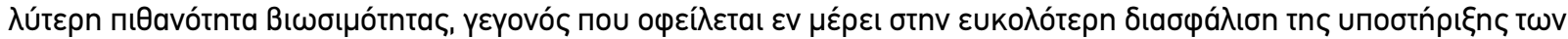

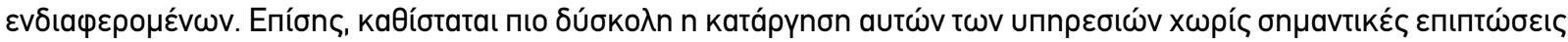

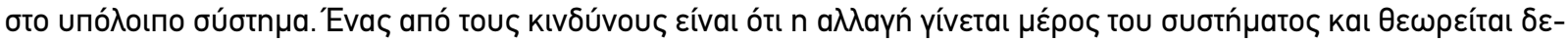

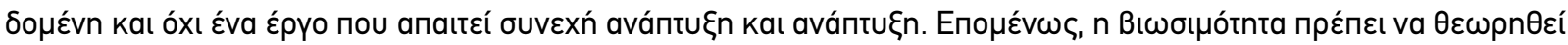

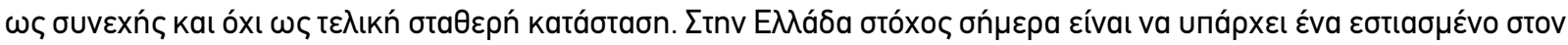

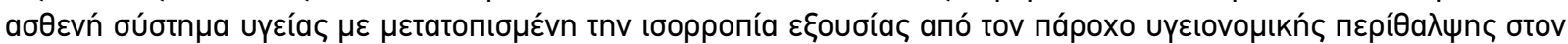

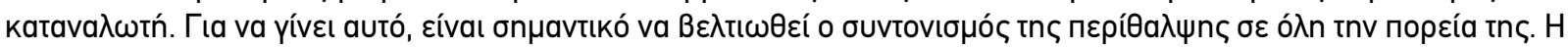

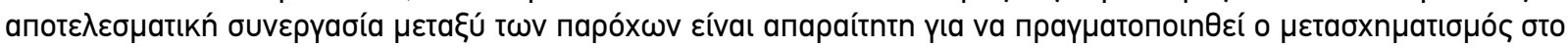

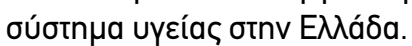

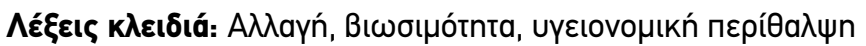

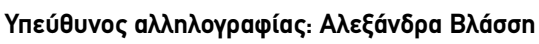

e-mail:alessandra_vlassi@yahoo.gr

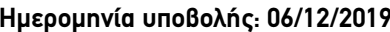

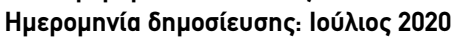

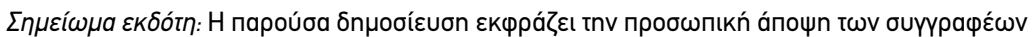

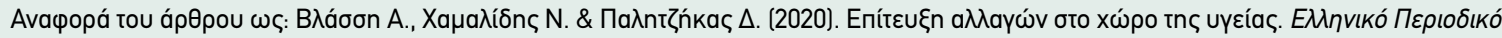

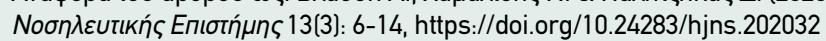




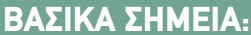

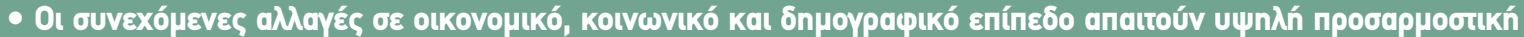

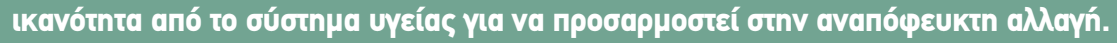

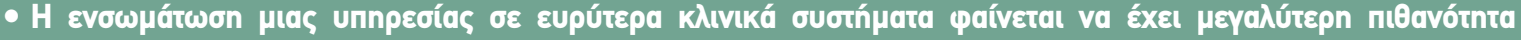
Bicuotpótntas.

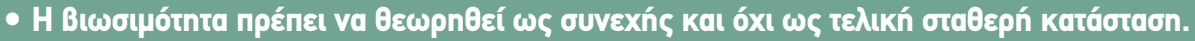

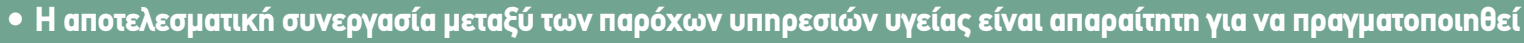

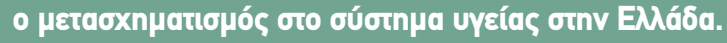

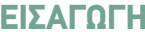

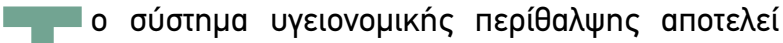

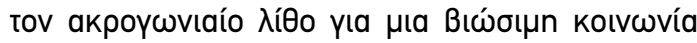

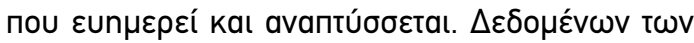

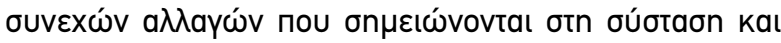

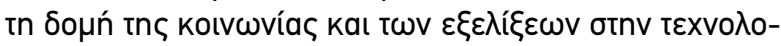

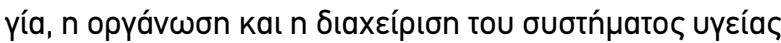

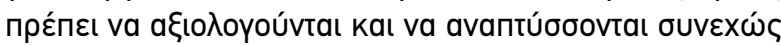

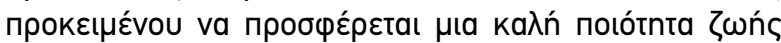

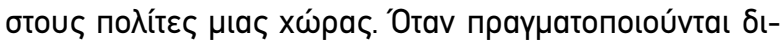

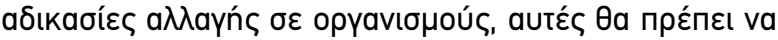

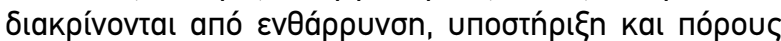

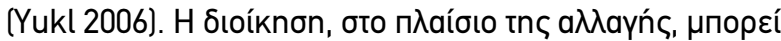

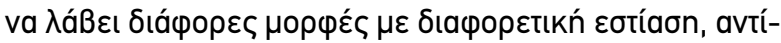

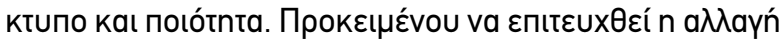

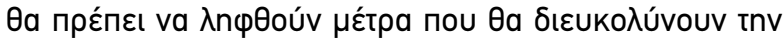

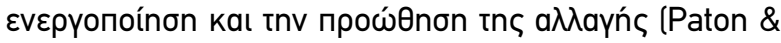

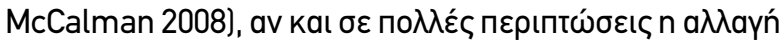

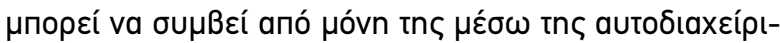
onc otnv opyávwon (Yukl 2006).

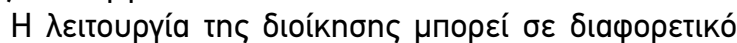

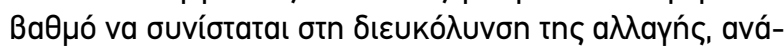

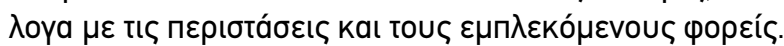

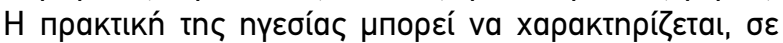

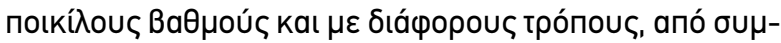

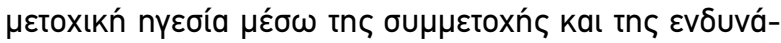

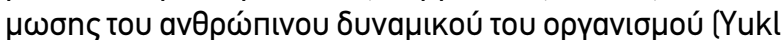

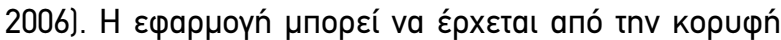

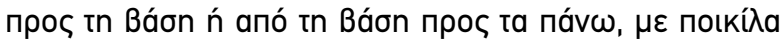

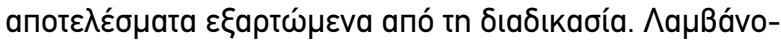

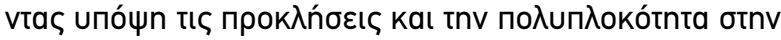

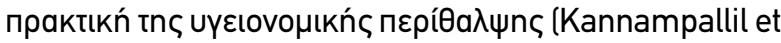

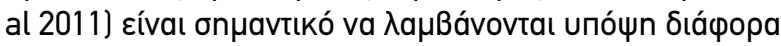

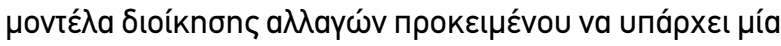
бфаıрıкń проoпtıkń.

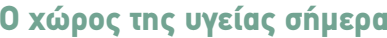

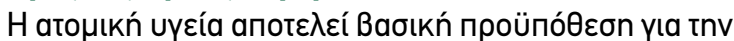

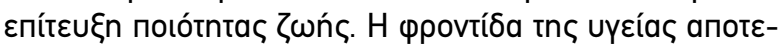

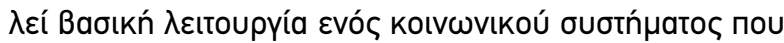

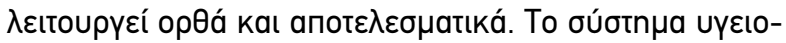

vo

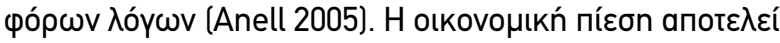

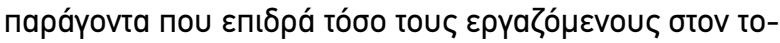

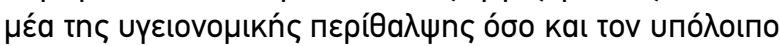

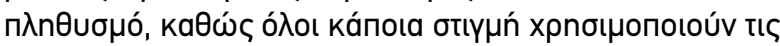

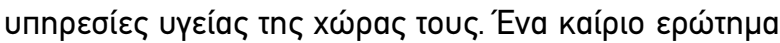

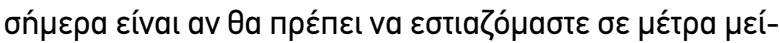

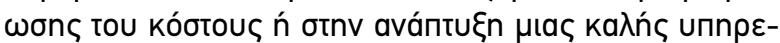

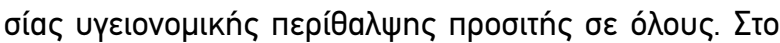

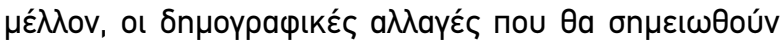

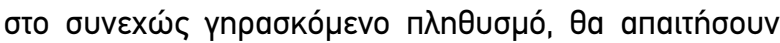

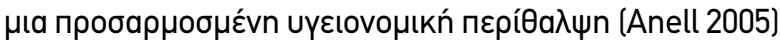

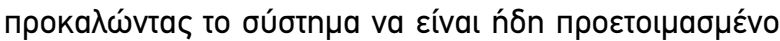

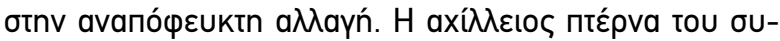

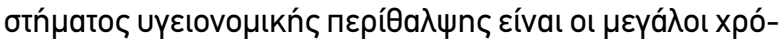

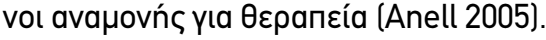

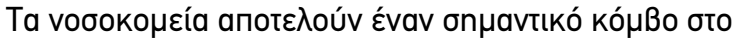

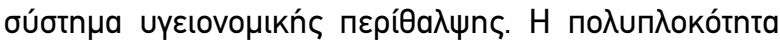

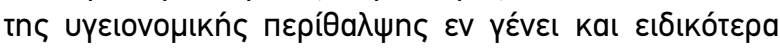

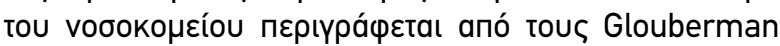

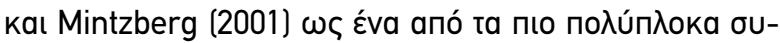

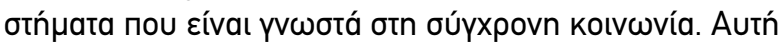

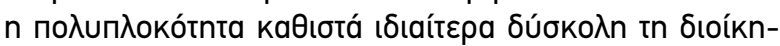

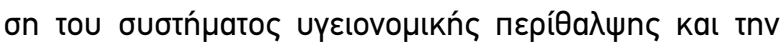

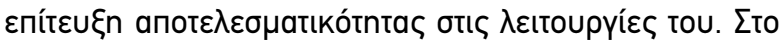

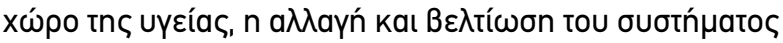

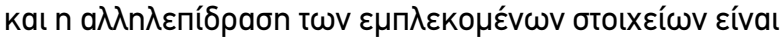

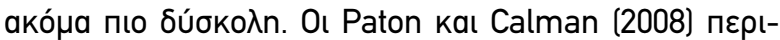

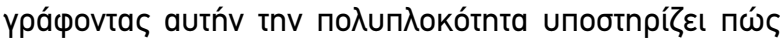

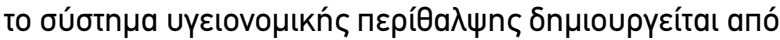

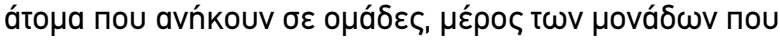

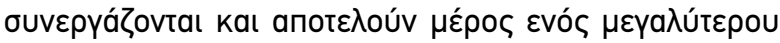

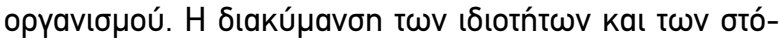

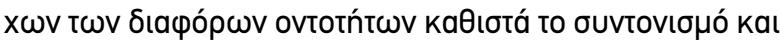

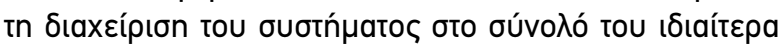

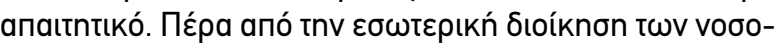

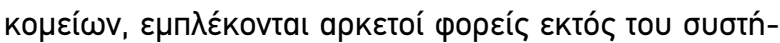

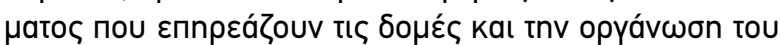
xẃpou tnc uyzías.

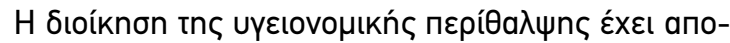

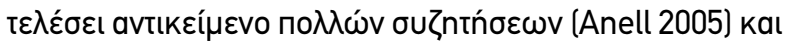




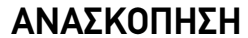

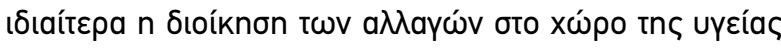

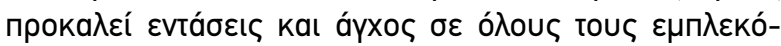

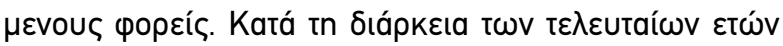

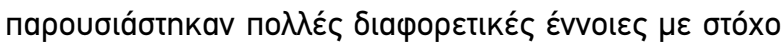

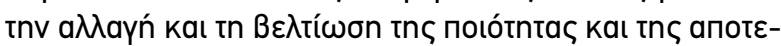

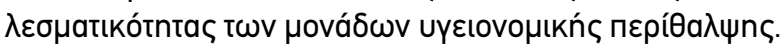

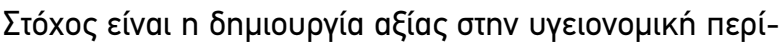

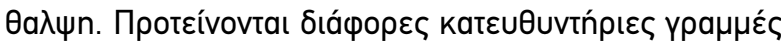

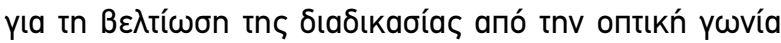

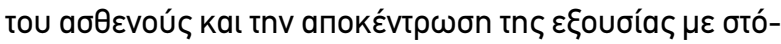

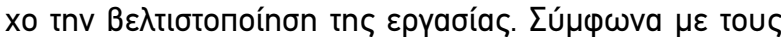

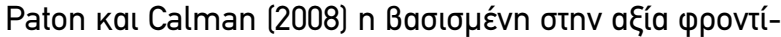

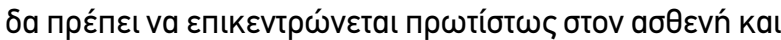

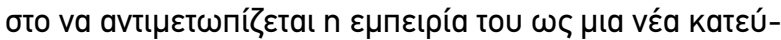

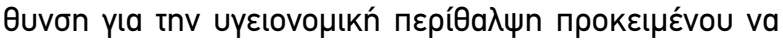

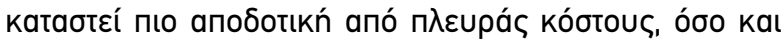

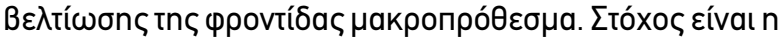

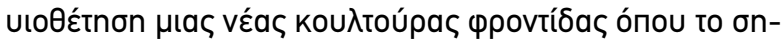

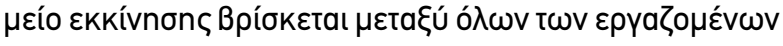

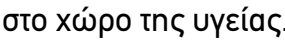

\section{Opyaviouoí uүદíą̧ kat avántuछ̨n}

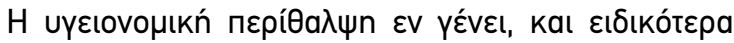

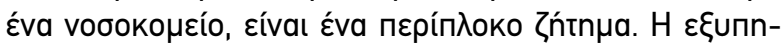

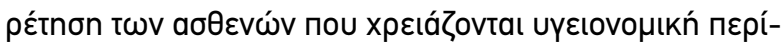

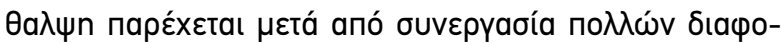

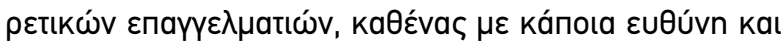

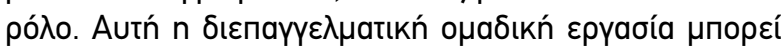

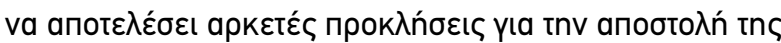

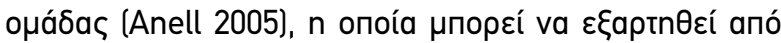

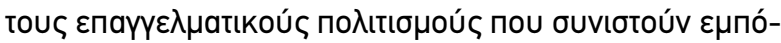

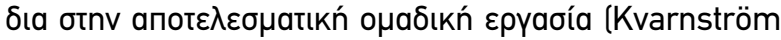

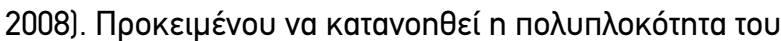

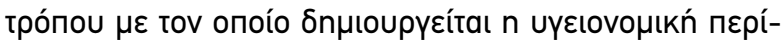

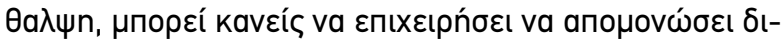
á

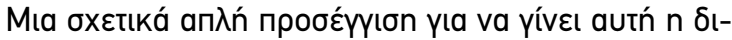
aípeon үívetaı anó touç Glouberman \& Mintzberg (2001).

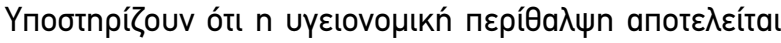

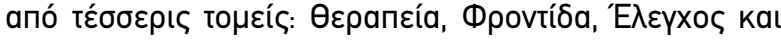

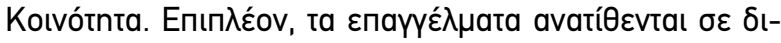

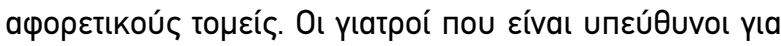

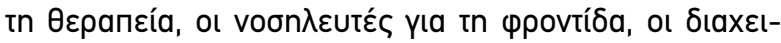

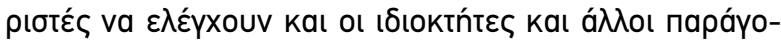

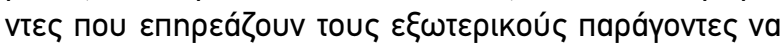

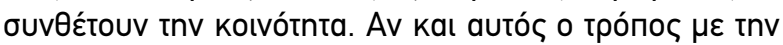

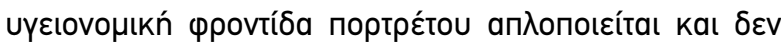

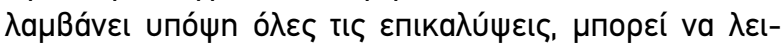

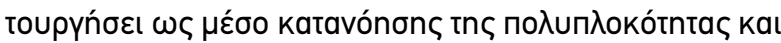

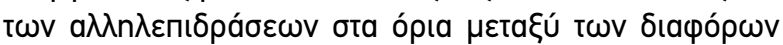

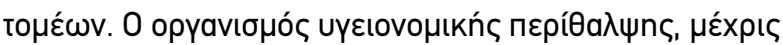

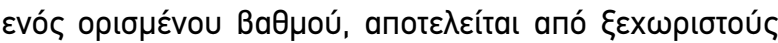

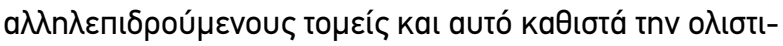

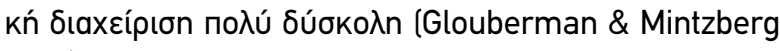

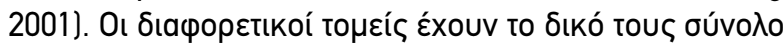

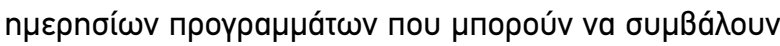

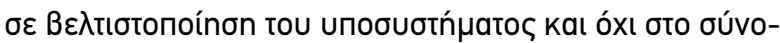

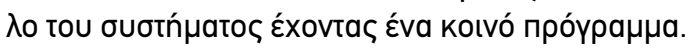

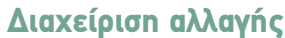

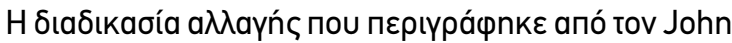

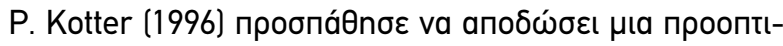

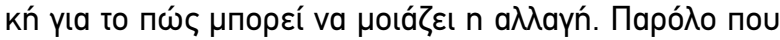

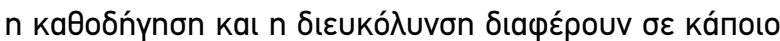

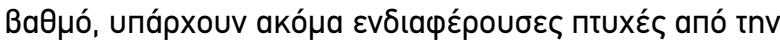

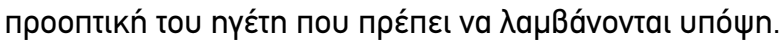

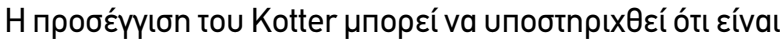

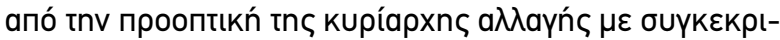

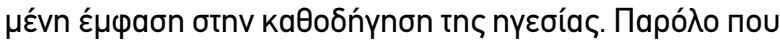

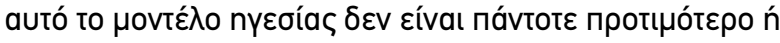

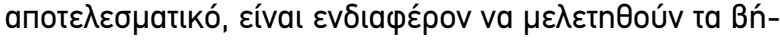

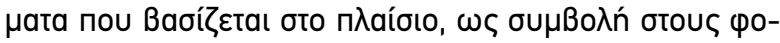

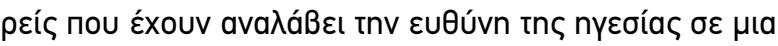

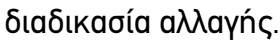

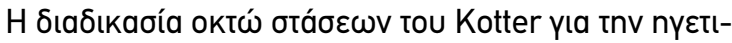

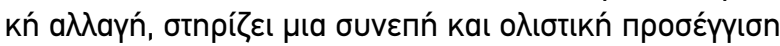

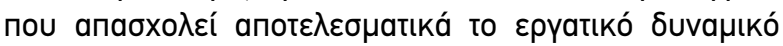
(Kotter 1996).

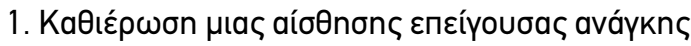

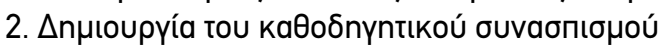

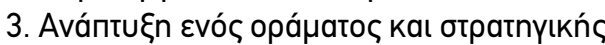

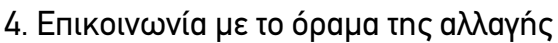

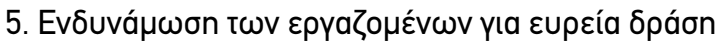

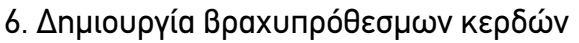

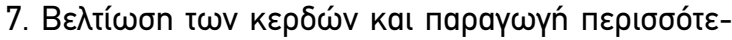
$\rho \omega v a \lambda \lambda a \gamma \omega ́ v$

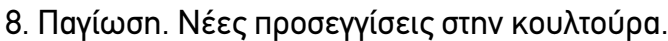

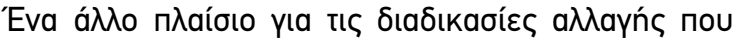

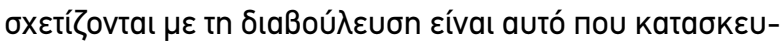

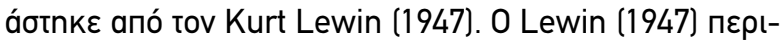

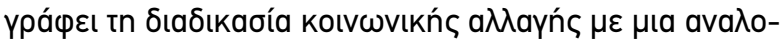

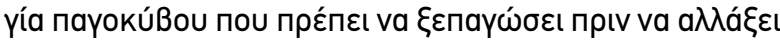

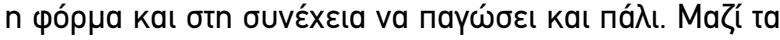

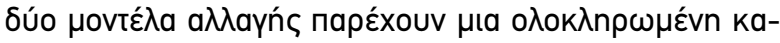

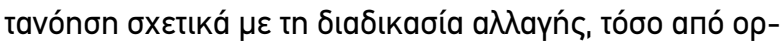

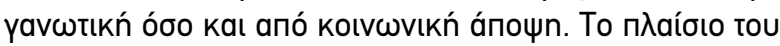

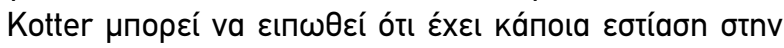

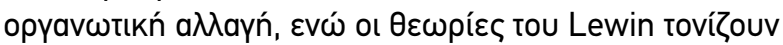

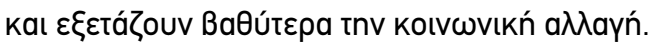

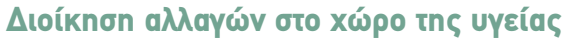

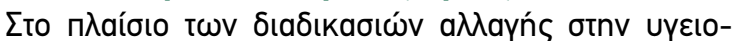

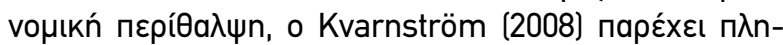

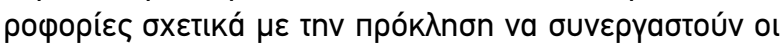

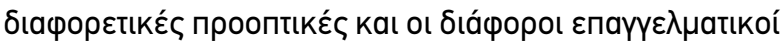

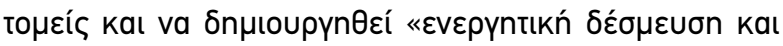




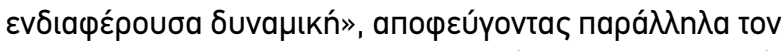

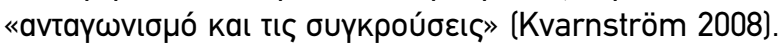

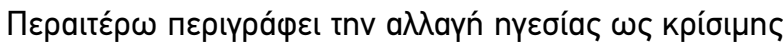

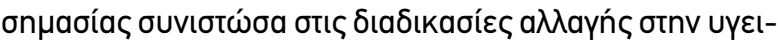

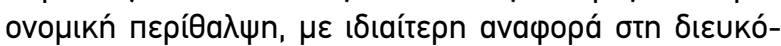

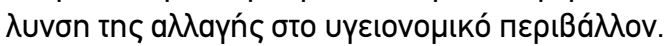

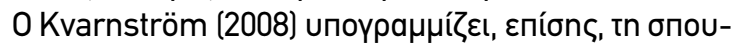

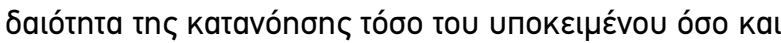

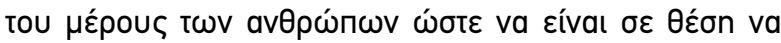

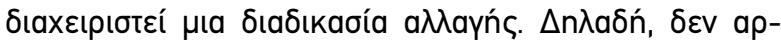

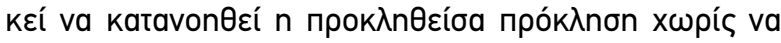

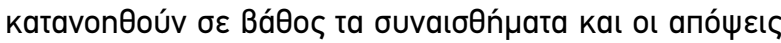

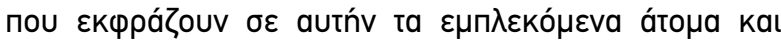

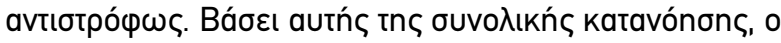

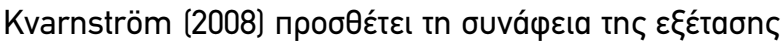

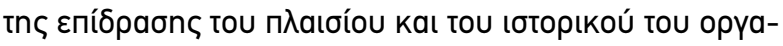

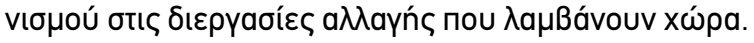

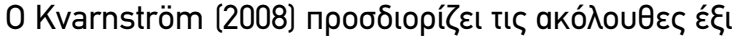

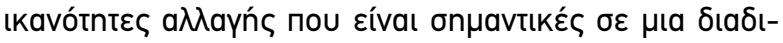

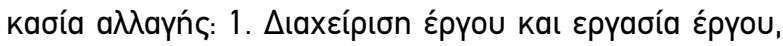

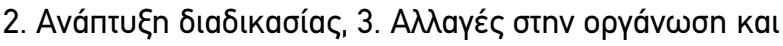

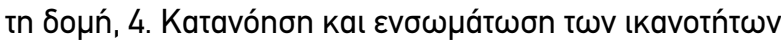

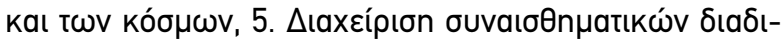

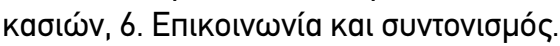

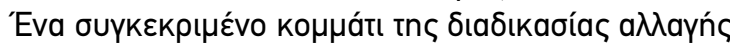

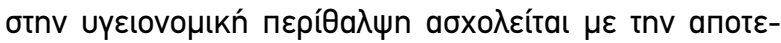

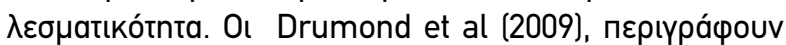

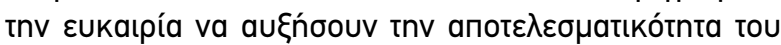

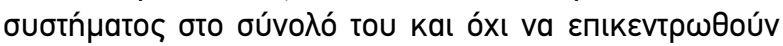

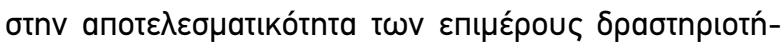

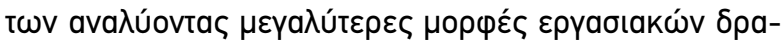

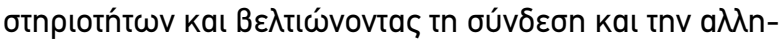

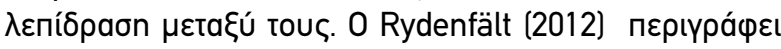

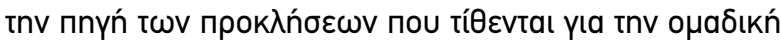

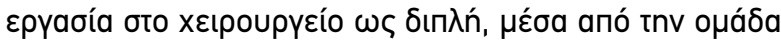

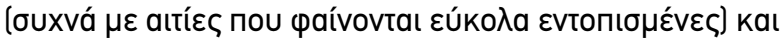

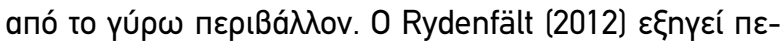

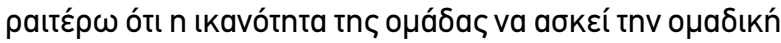

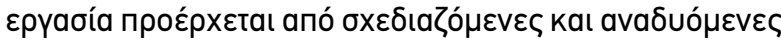

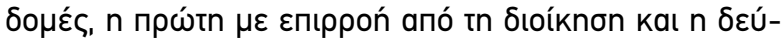

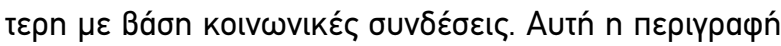

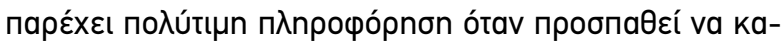

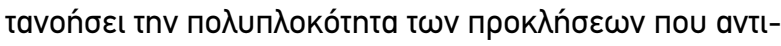

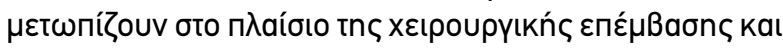

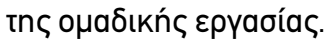

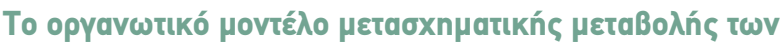

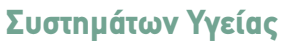

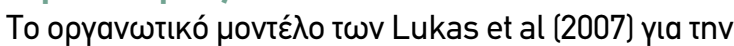

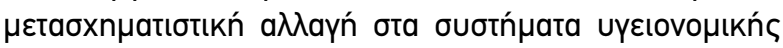

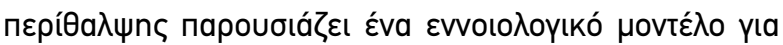

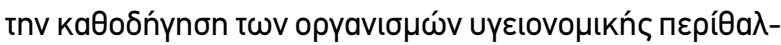

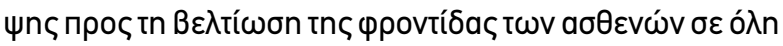

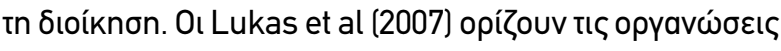

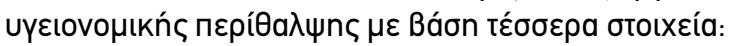

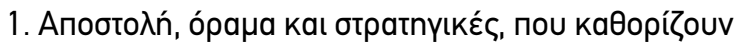

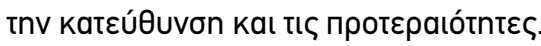

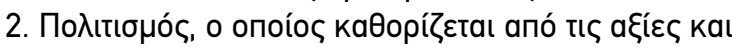
touৎ Kavóveৎ.

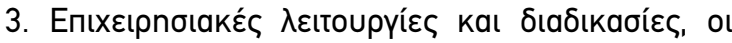

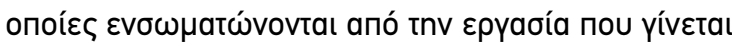

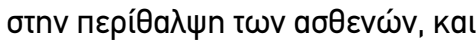

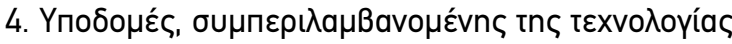

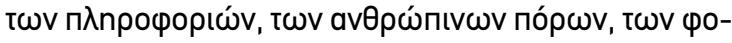

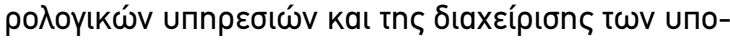

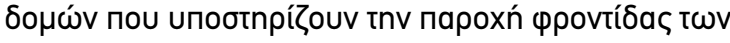

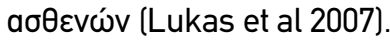

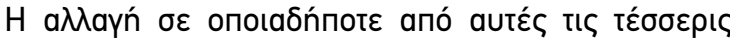

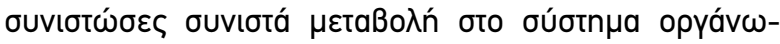

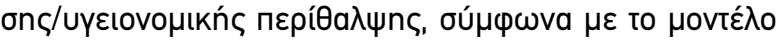

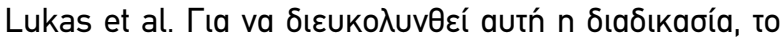

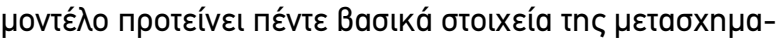

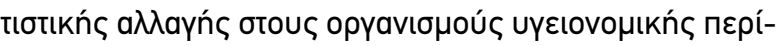
$\theta a \lambda \psi n c:$

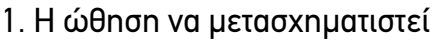

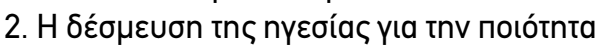

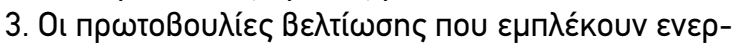

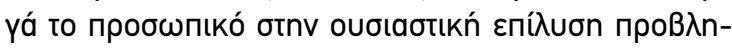

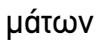

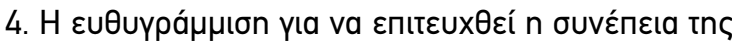
opyávwons.

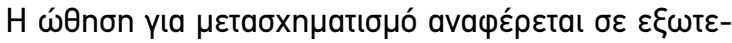

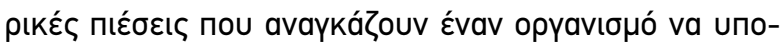

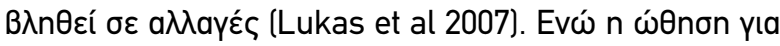

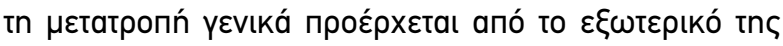

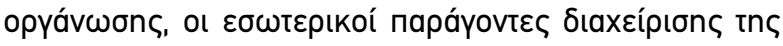

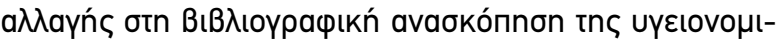

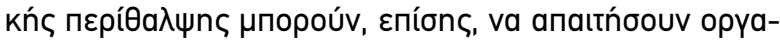

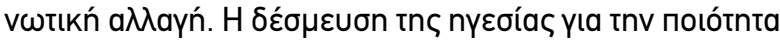

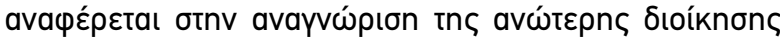

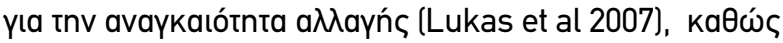

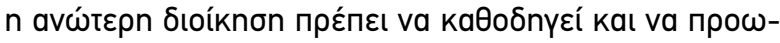

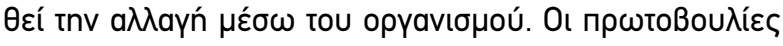

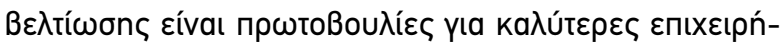

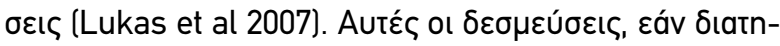

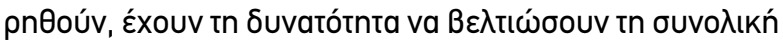

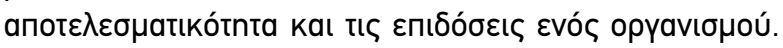

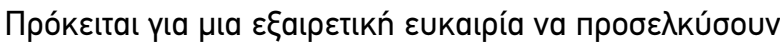

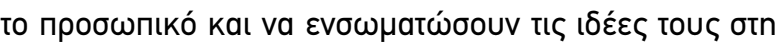

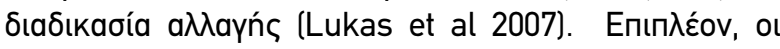

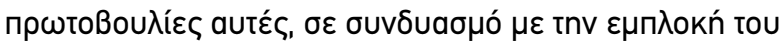

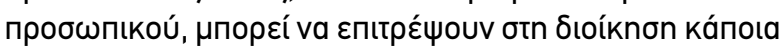

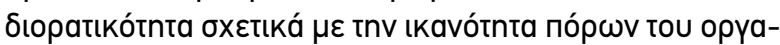

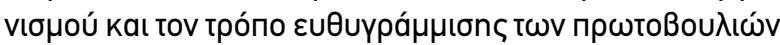

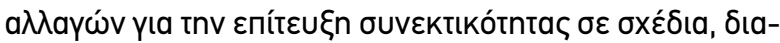

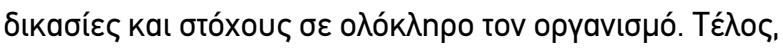




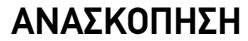

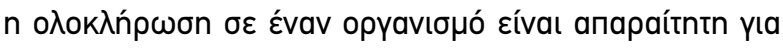

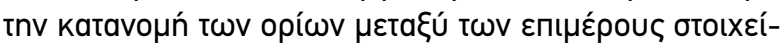

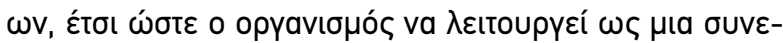

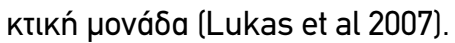

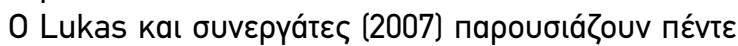

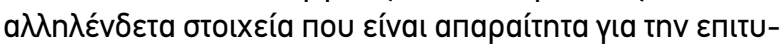

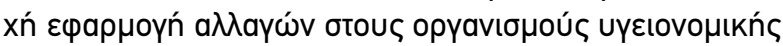

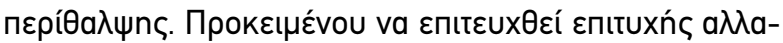

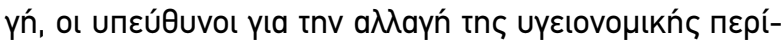

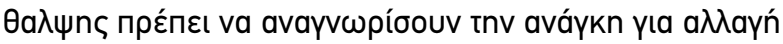

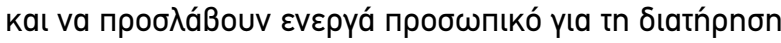

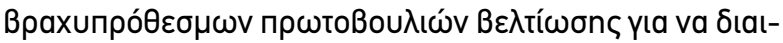

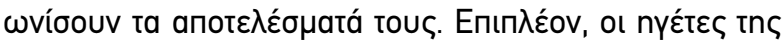

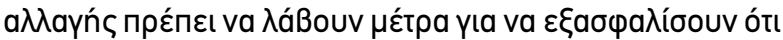

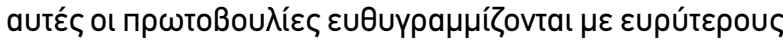

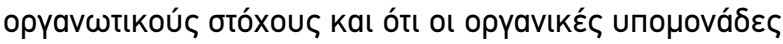

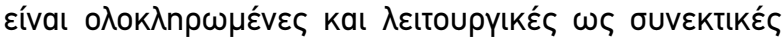

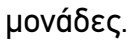

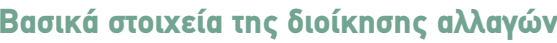

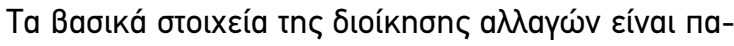

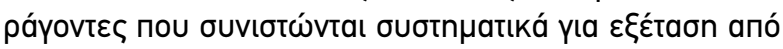

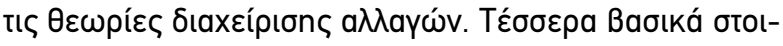

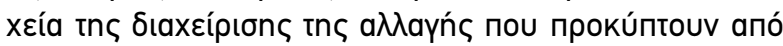

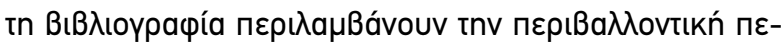

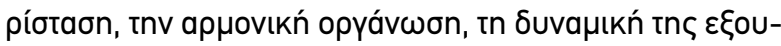
бías kal tnv opyavwtıkń ıkavótnta:

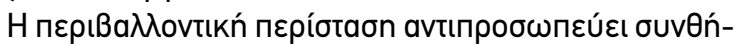

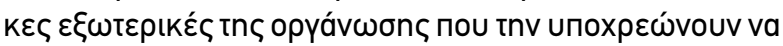

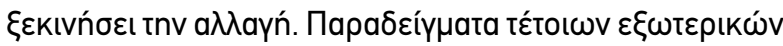

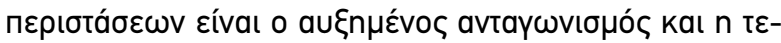

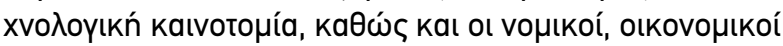

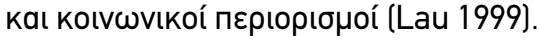

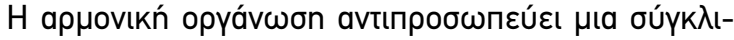

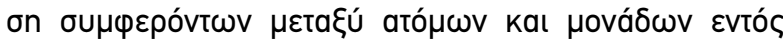

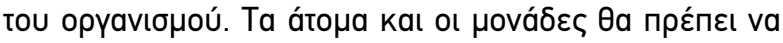

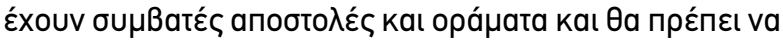

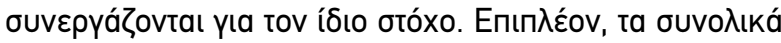

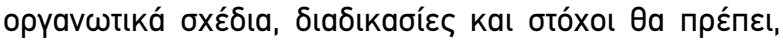

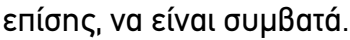

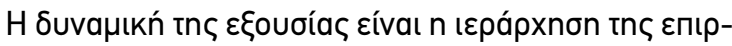

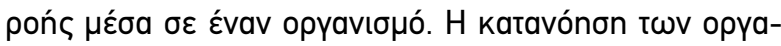

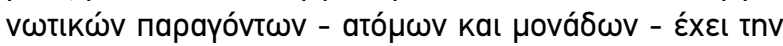

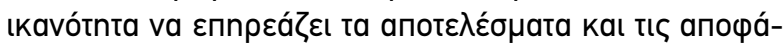

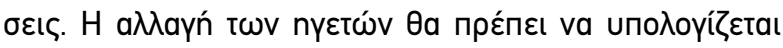

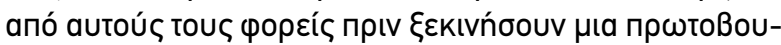

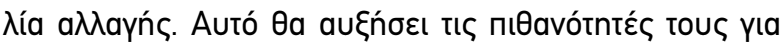

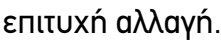

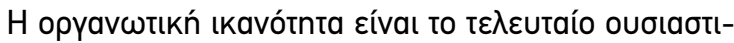

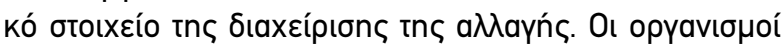

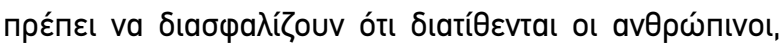

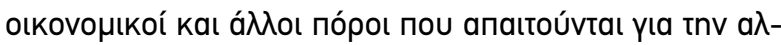

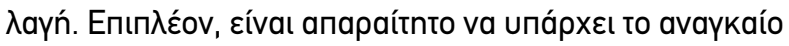

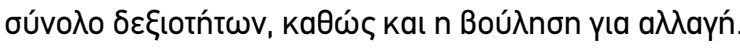

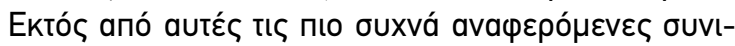

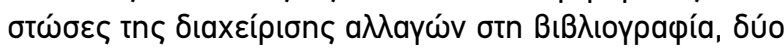

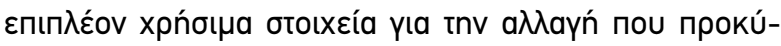

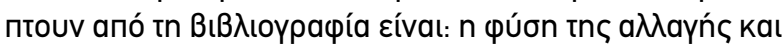

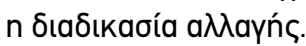

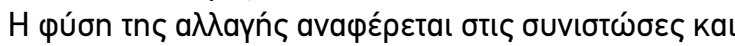

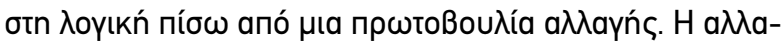

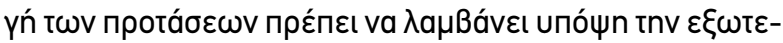

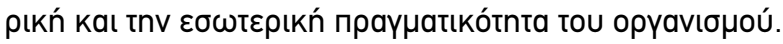

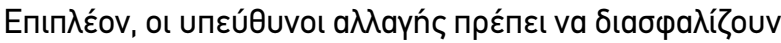

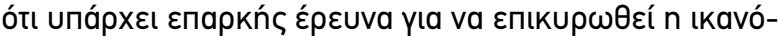

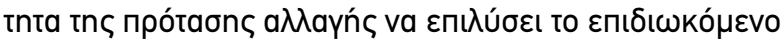

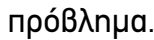

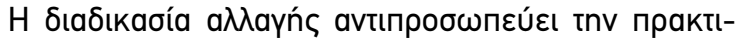

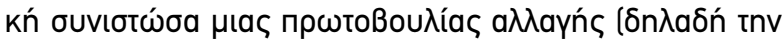

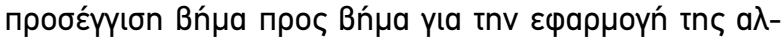

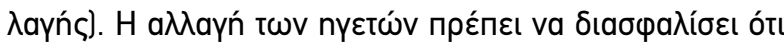

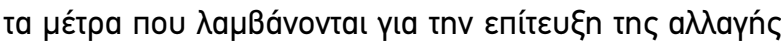

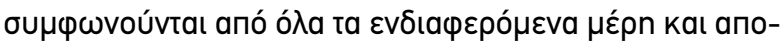

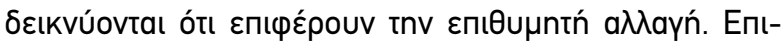

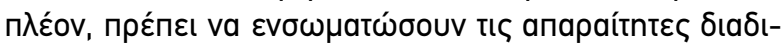

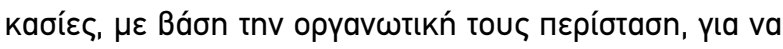
періopíoouv tnv avtíotaon.

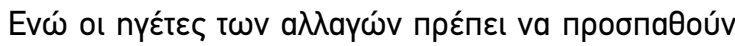

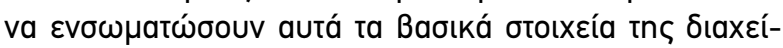

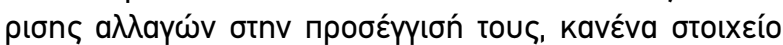

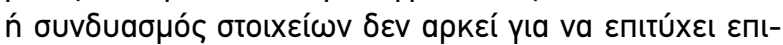

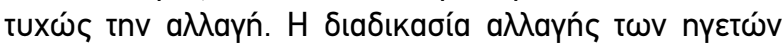

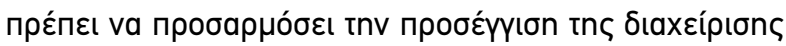

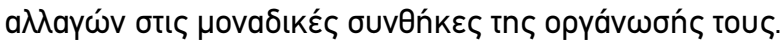

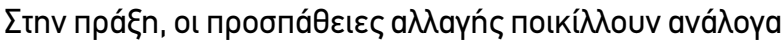

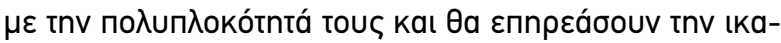

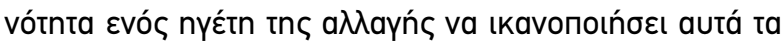

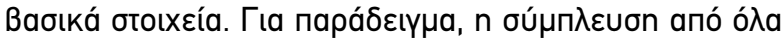
ta áto

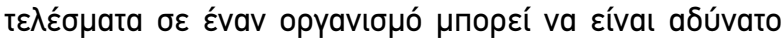

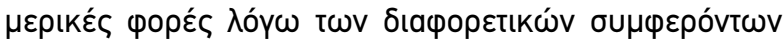

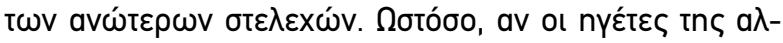

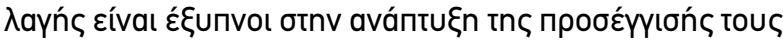

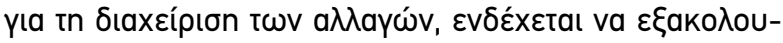

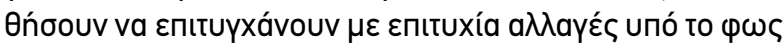

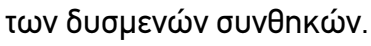

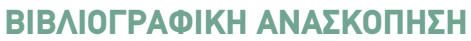

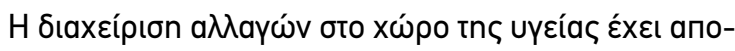

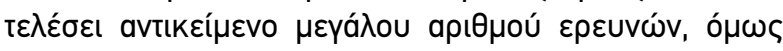

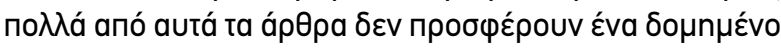

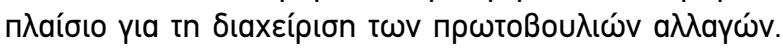

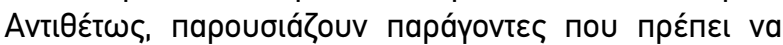

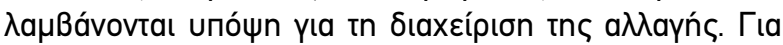

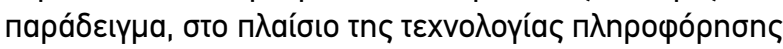

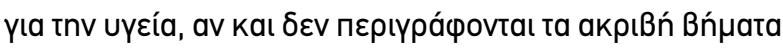




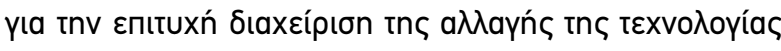

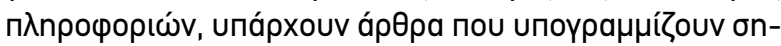

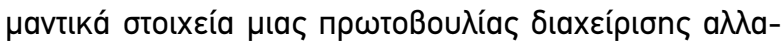

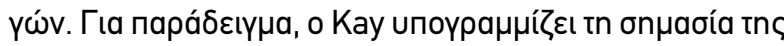

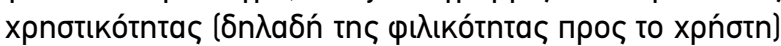

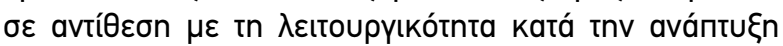

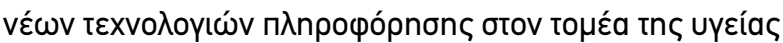
(Kay 2005).

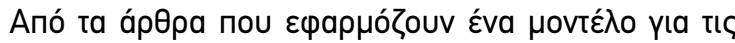

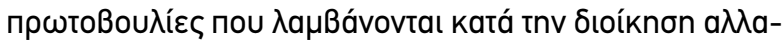

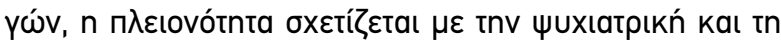

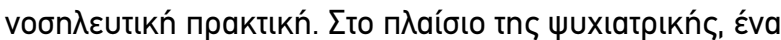

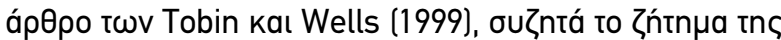

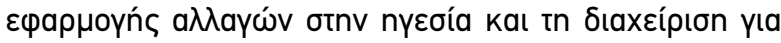

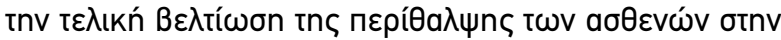

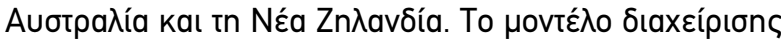

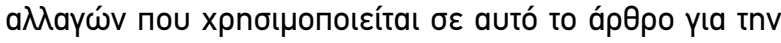

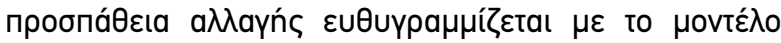

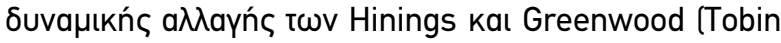
\& Wells 1999). ¿tnv aváduoń touৎ yıa tov трónо $\mu \varepsilon$ tov

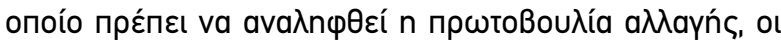

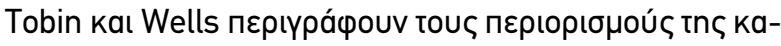

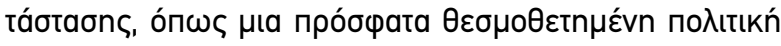

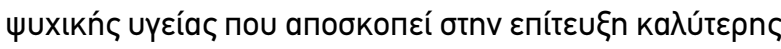

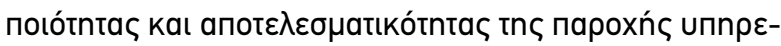

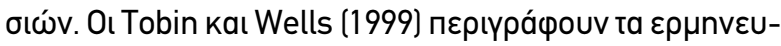

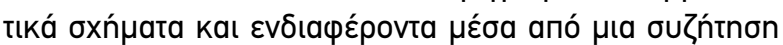

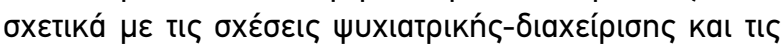

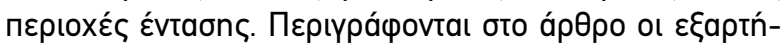

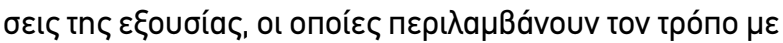

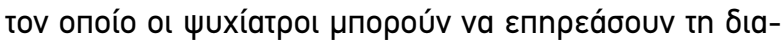

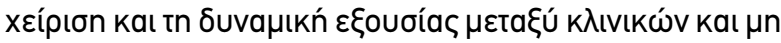

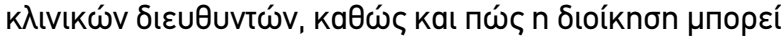

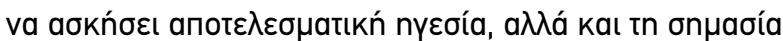

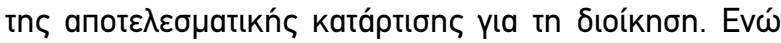

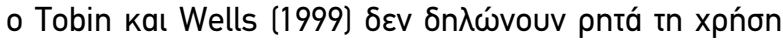

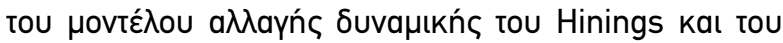

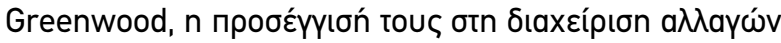

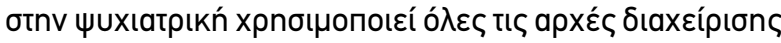

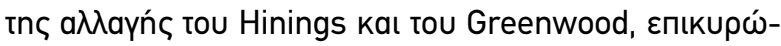

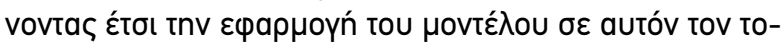

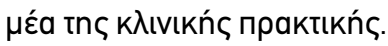

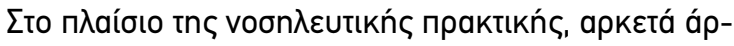

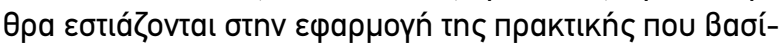

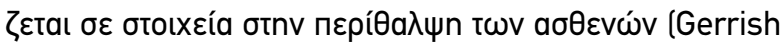

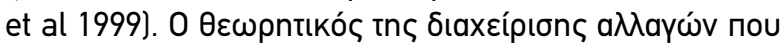

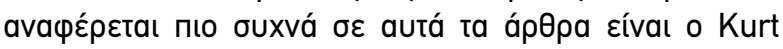

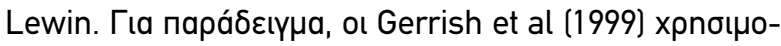

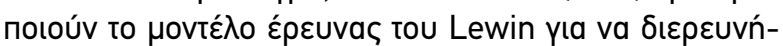

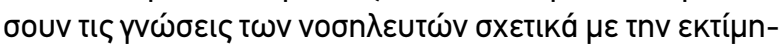

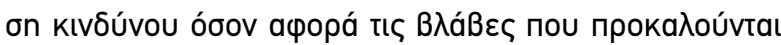

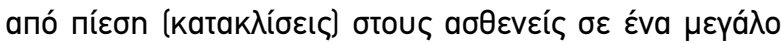

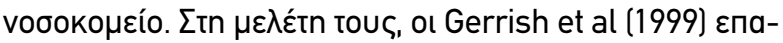

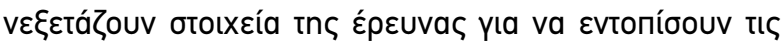

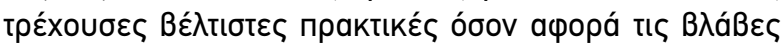

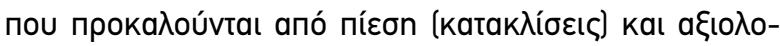

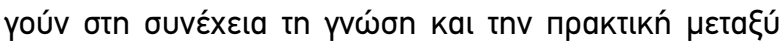

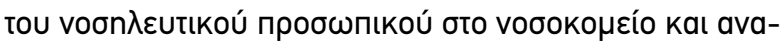

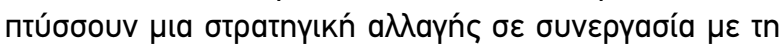

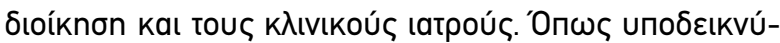

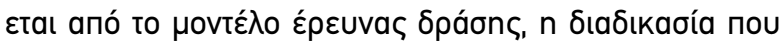

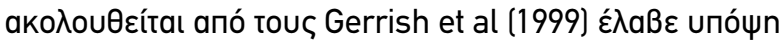

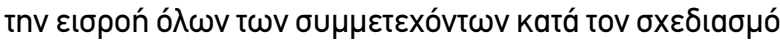

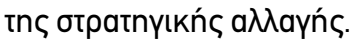

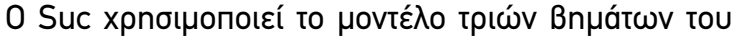

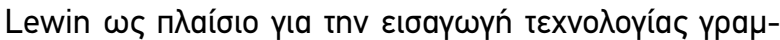

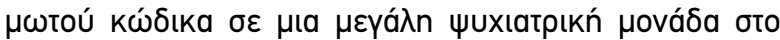

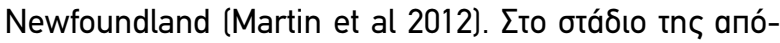

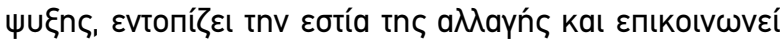

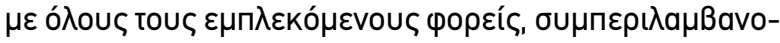

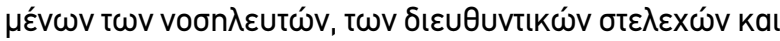

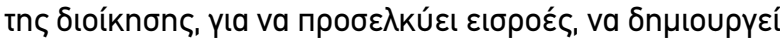

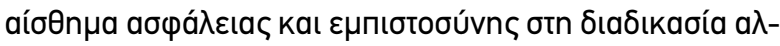

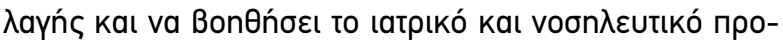

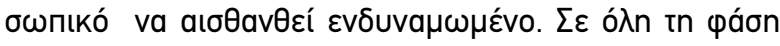

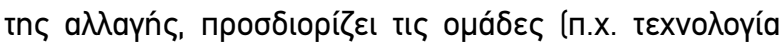

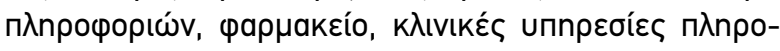

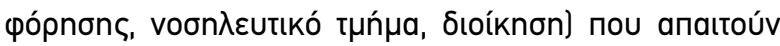

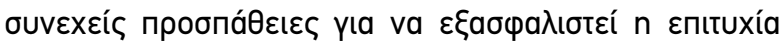

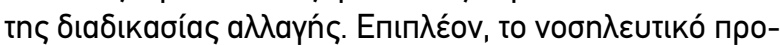

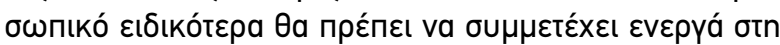

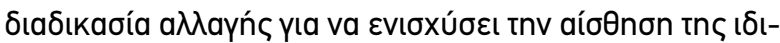

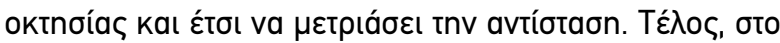

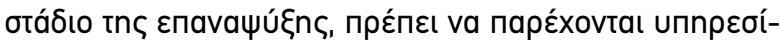

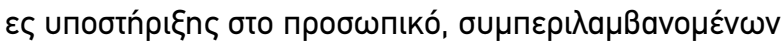

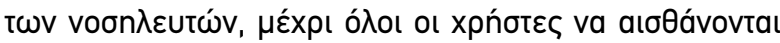

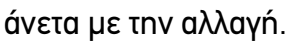

Oı Prokosch kaı n Ganslandt (2009) xрnoıнопоıоúv

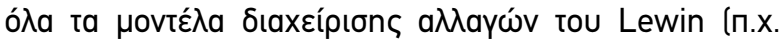

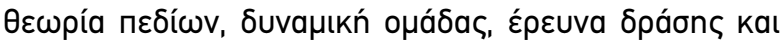

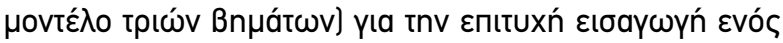

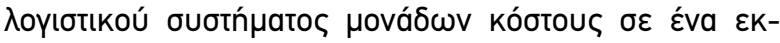

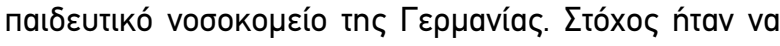

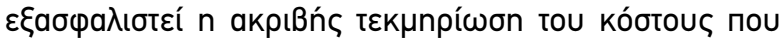

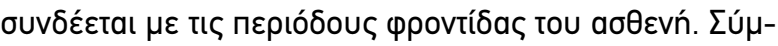

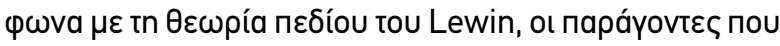

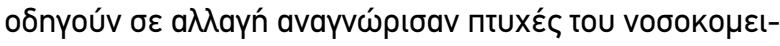

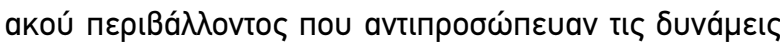

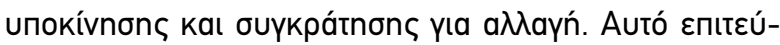

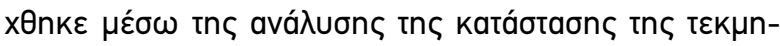

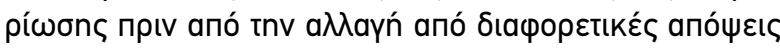

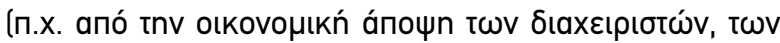

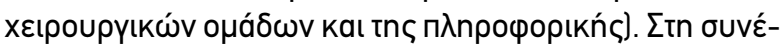

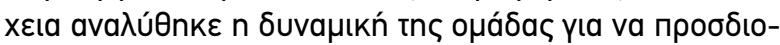

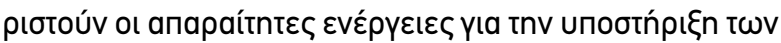

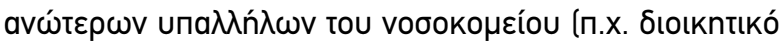




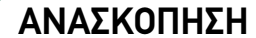

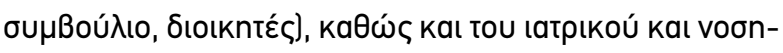

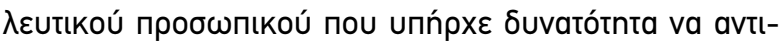

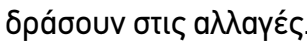

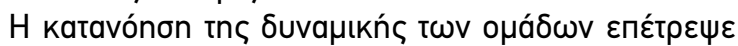

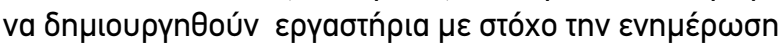

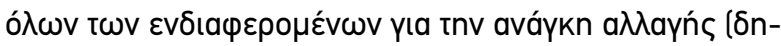

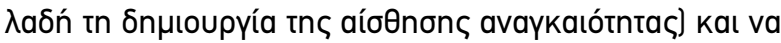

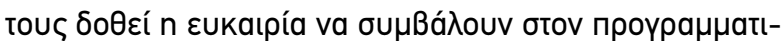

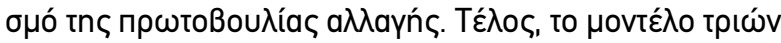

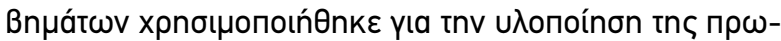

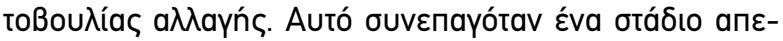

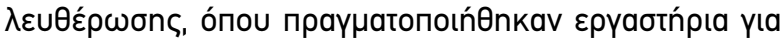

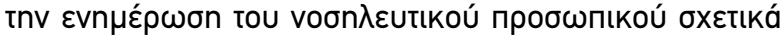

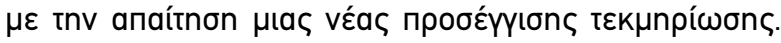

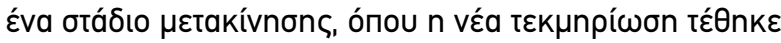

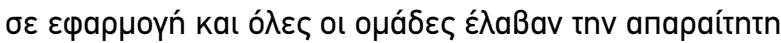

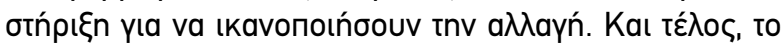

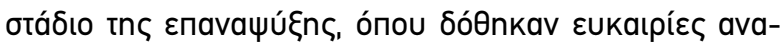

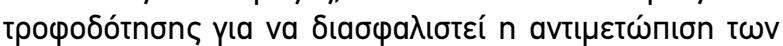

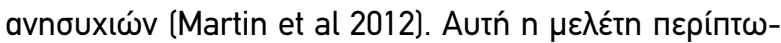

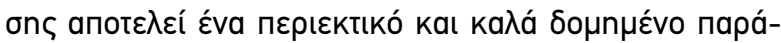

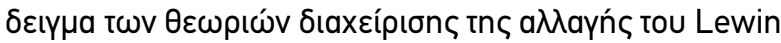

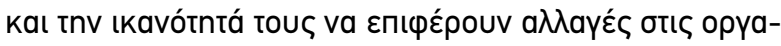

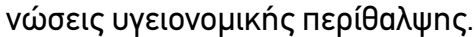

\section{¿YMПEPA¿MATA}

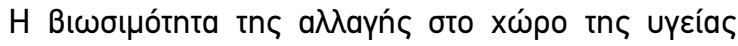

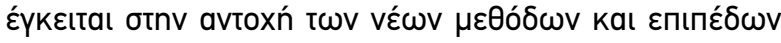

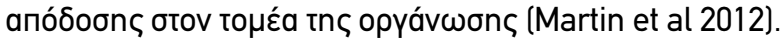

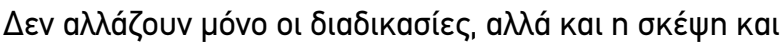

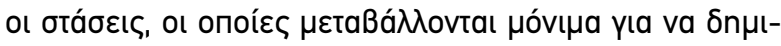

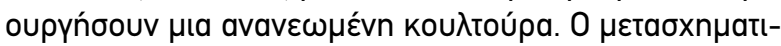

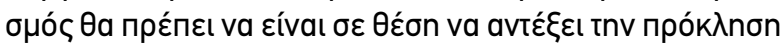

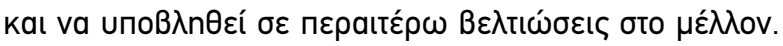

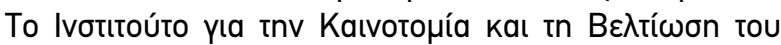

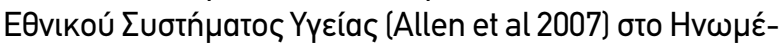

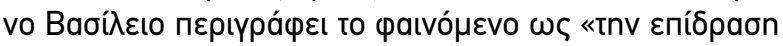

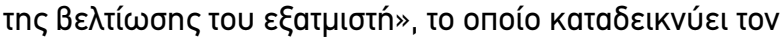

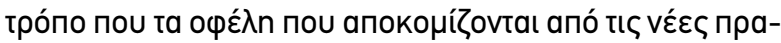

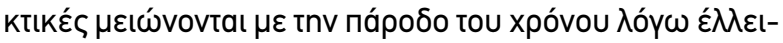

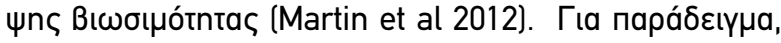

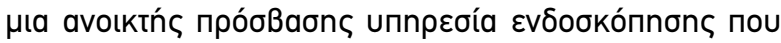

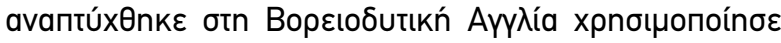

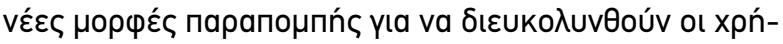

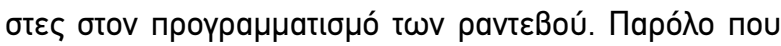

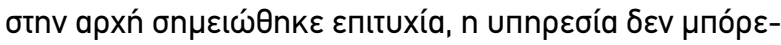

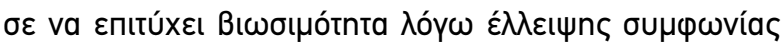

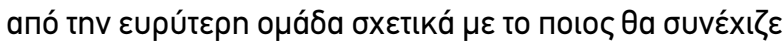

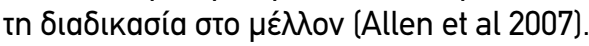

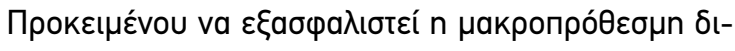

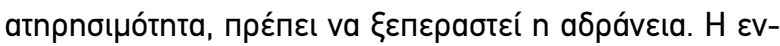

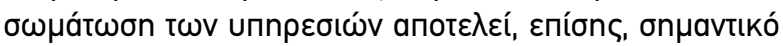

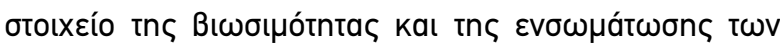

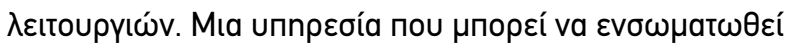

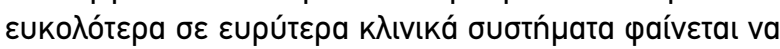

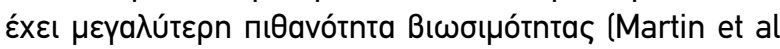

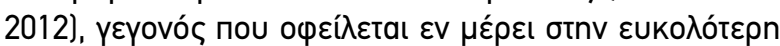

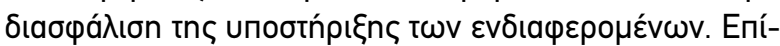

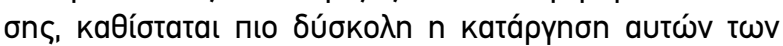

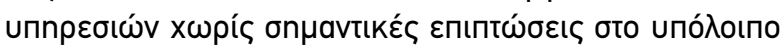

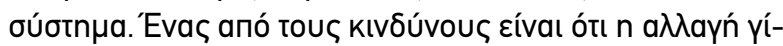

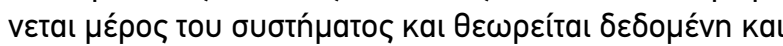
óx Bı

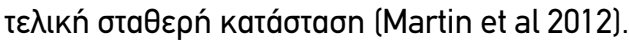

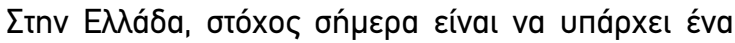

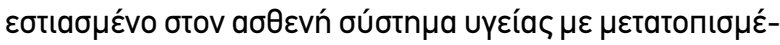

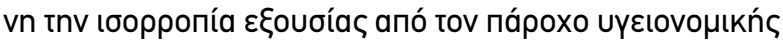

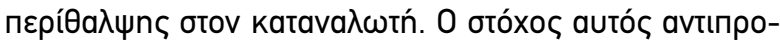

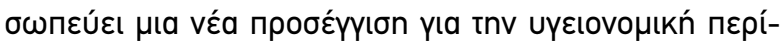

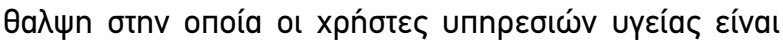

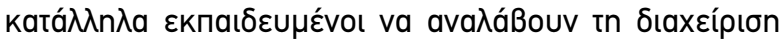

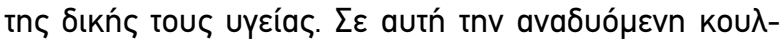

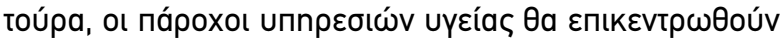

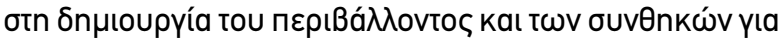

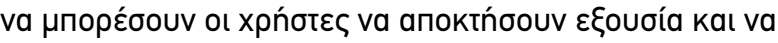

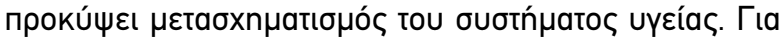

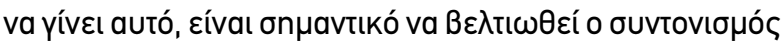

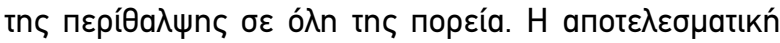

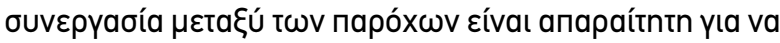

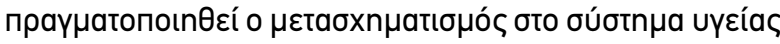

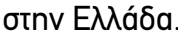

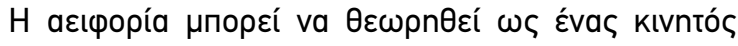

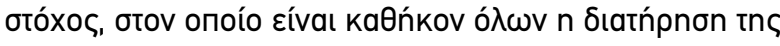

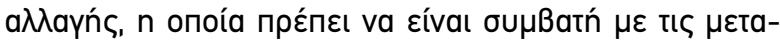

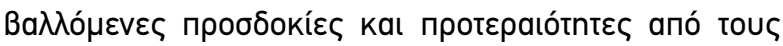

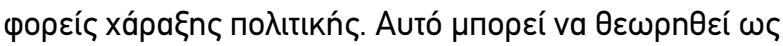

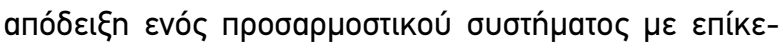

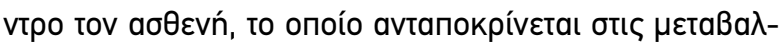

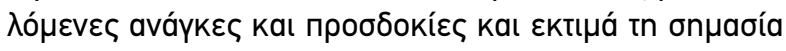

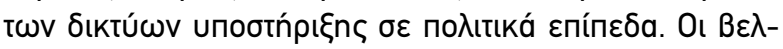

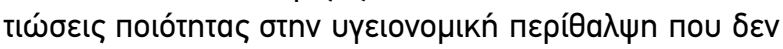

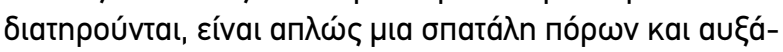

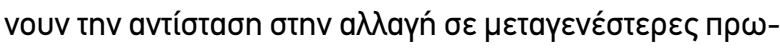

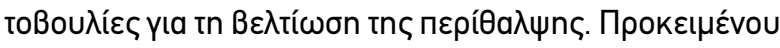

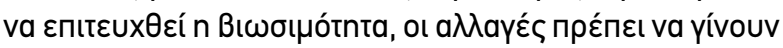

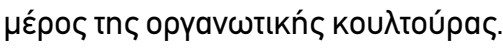




\section{ВIBАІОГРАФІА}

Allen C., Greenwood I., Hudson S., Ketley D., Maher L., Penny J. Rogers J., Singh J. Tibble J. \& Woodard F. for the NHS Institute for Innovation and Improvement. (2007). Innovation, N.I.f. and Improvement, Sustainability: And Its Relationship with Spread and Adoption. NHS Institute for Innovation and Improvement. University of Warwick. Coventry. UK.

Anell A. (2005). Swedish Healthcare under pressure. Health Economics 14: 237-254

Drummond-Hay R. \& Bamford $\Delta$. (2009). A case study into planning and change management within the UK National Health Service. International Journal of Public Sector Management 22(4): 324-337.

Gerrish K., Clayton J., Nolan M., Parker K. \& Morgan L. (1999). Promoting evidence-based practice: managing change in the assessment of pressure damage risk. Journal of Nursing Management 7(6): 355.

Glouberman S. \& Mintzberg H. (2001). Managing the Care of Health and the Cure of Disease - Part I: Differentiation. Healthcare Management Review 2: 56-69.

Kannampallil T.G., Schauer G.F., Cohen T. \& Patel V.L. (2011). Considering complexity in healthcare systems. Journal of Biomedical Informatics 44: 943-947.

Kay S. (2005). Usability: A critical success factor for managing change in the clinical infostructure. Informatics for Health and Social Care 30(2): 173-178.

Kotter J.P. (1996). Leading Change. Boston: Harvard Business School Press.

Kvarnström S. (2008). Difficulties in collaboration: A critical incident study of interprofessional healthcare teamwork. Journal of Interprofessional Care 22(2): 191-203.
Lau A.T.T. (1999). Making sense of contemporary strategic implementation: towards a conceptual model. Public Administration \& Management: An Interactive Journal 4(4): 494507.

Lewin K. (1947). Frontiers in Group Dynamics: Concept, Method and Reality in Social Science; Social Equilibria and Social Change. Human Relations, 1(1): 5-41.

Lukas C.V., Holmes S.K., Cohen A.B., Restuccia J., Cramer I.E., Shwartz M., Charns M.P. (2007). Transformational change in health care systems: an organizational model. Health care management review 32(4): 309-320.

Martin G.P., Weaver S., Currie G., Finn R. \& McDonald R. (2012) Innovation sustainability in challenging health-care contexts: embedding clinically led change in routine practice. Health Services Management Research 25(4): 190-199.

Paton R.A. \& McCalman J. (2008). Change Management: A Guide to Effective Implementation. Third Edition. London: SAGE Publications Ltd.

Rydenfält C. (2012). Social structures in the operating room: how contradicting rationalities and trust affect work. Journal of Advanced Nursing 68(4): 783-795.

Šuc J., Prokosch H. \& Ganslandt T. (2009). Applicability of Lewins change management model in a hospital setting. Methods Inf Med 48: 419-428.

Tobin M. \& Wells J. (1999). Psychiatrists managing change: lost control or at a loss. Australasian Psychiatry 7(4): 194-198.

Yukl G. (2006). Leadership in Organisations. Sixth Edition. New Yersey: Pearson Education. 


\title{
Achieving change in the Healthcare Sector
}

\author{
Alessandra Vlassi ${ }^{1}$, Nikolaos Chamalidis², Dimitrios Palitzikas ${ }^{3}$ \\ 1. Health Inspector, MSc, PhD(c), YPEDYFKA-EOPYY \\ 2. Administrative Employee, MSc, EOPY, Thessaloniki \\ 3. RN, MSc, G.Papanikolaou General Hospital
}

\section{ABSTRACT}

Individual health is an essential prerequisite for achieving quality of life. Healthcare is a basic function of a well-functioning social system. Contemporary changes at economic, social and demographic level require healthcare to show a high adaptive capacity to adapt to the inevitable change. Healthcare change processes need to involve different professional sectors and create an active commitment while avoiding competition and conflicts between the parties involved. In order to ensure long-term sustainability in change, inertia must be overcome. Integration of services is also an important element of sustainability and integration of functions. A service that can be more easily integrated into wider clinical systems seems to have a higher probability of sustainability, partly due to easier support from the stakeholders. It also makes it more difficult to remove these services without having a significant impact on the rest of the system. One of the dangers is that the change can become a part of the system and it is considered for granted and not as a project that requires constant development. Therefore, sustainability should be considered as a constantly evolving rather than a final steady state. In Greece, the goal today is to have a patient-centered health care system with a shifted balance of power from the healthcare provider to the consumer. In order to achieve this, it is important to improve the coordination throughout the healthcare system. Effective co-operation between providers is essential to transform the healthcare system in Greece.

Keywords: Healthcare, change, sustainability. 